Groups Geom. Dyn. 7 (2013), 961-976

DOI $10.4171 / \mathrm{GGD} / 212$
Groups, Geometry, and Dynamics

(C) European Mathematical Society

\title{
Hyperbolic hydra
}

\author{
Noel Brady, Will Dison and Timothy R. Riley*
}

\begin{abstract}
We give examples of hyperbolic groups with finite rank free subgroups of huge (Ackermannian) distortion.
\end{abstract}

Mathematics Subject Classification (2010). 20F65, 20F10, $20 \mathrm{~F} 67$.

Keywords. Hyperbolic group, subgroup distortion, hydra, Ackermann's function.

\section{Introduction}

1.1. Our result. Hyperbolic groups are algorithmically tractable (their word and conjugacy problems are straightforward) and are characterised by a tree-like property that geodesic triangles in their Cayley graphs are close to tripods [5], [8]. The purpose of this article is to show that nonetheless some harbour extreme wildness within their subgroups - their finite rank free subgroups, even. We prove (the terminology is explained below):

Theorem 1.1. There are hyperbolic groups $\Gamma_{k}$ for all $k \geq 1$ with free rank $(k+18)$ subgroups $\Lambda_{k}$ whose distortion satisfies $\operatorname{Dist}_{\Lambda_{k}}^{\Gamma_{k}} \succeq A_{k}$, that is, grows at least like the $k$-th of Ackermann's functions.

A distortion function $\operatorname{Dist}_{H}^{G}$ measures the degree to which a subgroup $H \leq G$ folds in on itself within $G$ by comparing the intrinsic word metric on $H$ with the extrinsic word metric inherited from $G$. Suppose that $S$ and $T$ are finite generating sets for $G$ and $H$, respectively. Then

$$
\operatorname{Dist}_{H}^{G}(n):=\max \left\{d_{T}(1, g) \mid g \in H \text { with } d_{S}(1, g) \leq n\right\} .
$$

Up to the following equivalence, capturing qualitative agreement of growth rates, Dist $_{H}^{G}$ does not depend on $S$ and $T$. For $f, g: \mathbb{N} \rightarrow \mathbb{N}$, we write $f \preceq g$ when there

*The first author gratefully acknowledges partial support from NSF grant DMS-0906962 and the third author from NSF grant DMS-1101651 and Simons Foundation Collaboration Grant 208567. 
exists $C>0$ such that $f(n) \leq C g(C n+C)+C n+C$ for all $n$. Define $f \simeq g$ when $f \preceq g$ and $g \preceq f$.

Ackermann's $A_{k}: \mathbb{N} \rightarrow \mathbb{N}$ are a family of fast growing functions defined recursively:

$$
\begin{aligned}
& A_{0}(n)=n+2 \quad \text { for } n \geq 0, \\
& A_{k}(0)= \begin{cases}0 & \text { for } k=1 \\
1 & \text { for } k \geq 2\end{cases}
\end{aligned}
$$

and

$$
A_{k+1}(n+1)=A_{k}\left(A_{k+1}(n)\right) \text { for } k, n \geq 0 .
$$

In particular, $A_{1}(n)=2 n, A_{2}(n)=2^{n}$ and $A_{3}(n)$ is the $n$-fold iterated power of 2. They are representatives of the successive levels of the Grzegorczyk hierarchy of primitive recursive functions - see, for example, [15].

1.2. The organisation of this article and an outline of our approach. Our groups $\Gamma_{k}$ are elaborations of the hydra groups

$$
\left.\left.G_{k}=\left\langle a_{1}, \ldots, a_{k}, t\right| t^{-1} a_{1} t=a_{1}, t^{-1} a_{i} t=a_{i} a_{i-1} \text { (for all } i>1\right)\right\rangle
$$

explored by the second and third authors in [6]. These $G_{k}$ are CAT(0), free-bycyclic, biautomatic, and can be presented with only one relator, and yet the subgroups $H_{k}:=\left\langle a_{1} t, \ldots, a_{k} t\right\rangle$ are free of rank $k$ and their distortion grows like the $k$-th of Ackermann's functions: Dist $_{H_{k}}^{G_{k}} \simeq A_{k}$.

This extreme distortion stems from a phenomenon which can be described as a re-imagining of Hercules' battle with the Lernaean Hydra. A hydra is a positive word $w$ on the alphabet $a_{1}, a_{2}, \ldots$. Hercules removes the first letter and then the creature regenerates in that each remaining $a_{i}$ with $i>1$ becomes $a_{i} a_{i-1}$. (Each remaining $a_{1}$ is unaffected.) This repeats and Hercules triumphs when the hydra is reduced to the empty word $\varepsilon$. The number of steps is denoted $\mathscr{H}(w)$. (Each step encompasses the removal of the first letter and then regeneration.) For example, $\mathscr{H}\left(a_{2}^{3}\right)=7$ :

$$
a_{2}^{3} \rightarrow\left(a_{2} a_{1}\right)^{2} \rightarrow a_{1} a_{2} a_{1}^{2} \rightarrow a_{2} a_{1}^{3} \rightarrow a_{1}^{3} \rightarrow a_{1}^{2} \rightarrow a_{1} \rightarrow \varepsilon .
$$

In [6] it is shown that Hercules will be victorious whatever hydra he faces, but the number of strikes it takes can be huge: the functions $\mathscr{H}_{k}$, defined by $\mathscr{H}_{k}(n)=\mathscr{H}\left(a_{k}^{n}\right)$, grow like Ackermann's functions: $\mathscr{H}_{k} \simeq A_{k}$.

The group $G_{k}$ is not hyperbolic because it has the subgroup $\left\langle a_{1}, t\right\rangle \cong \mathbb{Z}^{2}$. We obtain $\Gamma_{k}$ by combining $G_{k}$ with another free-by-cyclic group, which is hyperbolic, in such a way that the hydra phenomenon persists in $\Gamma_{k}$, but the troublesome "Euclidean" relations $t^{-1} a_{1} t=a_{1}$ are replaced by something "hyperbolic".

In Section 2 we will give two presentations $P_{k}$ and $Q_{k}$ for $\Gamma_{k}$ and will prove they are equivalent. $P_{k}$ is well suited to proving hyperbolicity: the associated Cayley 
2-complex, suitably metrized, will be shown in Section 3 to be CAT(0) and to contain no isometrically embedded copies of $\mathbb{R}^{2}$ and so is hyperbolic by the Flat Plane Theorem. $Q_{k}$ places $\Gamma_{k}$ in a class of free-by-cyclic groups which we show in Section 4 (for $k \geq 2$ ) contain free subgroups of rank $k+18$ and distortion $\succeq A_{k}$. (In the case $k=1$, Theorem 1.1 is elementary: take $\Gamma_{1}$ to be a free group and $\Lambda_{1}$ to be $\Gamma_{1}$.)

1.3. Background. Other heavily distorted free subgroups of hyperbolic groups have been exhibited by Mitra [12]: for all $k$, he gives an example with a free subgroup of distortion like a $k$-fold iterated exponential function and, more extreme, an example where the number of iterations grows like $\log n$. Barnard, the first author and Dani developed Mitra's constructions into more explicit examples that are also CAT $(-1)$ [3]. We are not aware of any example of a hyperbolic group with a finite rank free subgroup of distortion exceeding that of our examples. Indeed, we do not know of a hyperbolic group with a finitely presented subgroup of greater distortion. The Rips construction, applied to a finitely presentable group with unsolvable word problem yields a hyperbolic (in fact, $C^{\prime}(1 / 6)$ small cancellation) group $G$ with a finitely generated subgroup $N$ such that $\operatorname{Dist}_{N}^{G}$ is not bounded above by any recursive function, but these $N$ are not finitely presentable. (See [1], §3.4, [7], Corollary 8.2, [9], §3, 3. $K_{3}^{\prime \prime}$, and [14].)

Whilst we will not call on it in this paper (as we will give the translation between the presentations $P_{k}$ and $Q_{k}$ explicitly), a result that lies behind how we came to our examples is that if a 2-complex admits an $S^{1}$-valued Morse function all of whose ascending and descending links are trees, then its fundamental group is free-by-cyclic [2]. (The ascending link for our examples is visible in Figure 2 as the subgraph made up of all edges connecting pairs of negative vertices. The descending is that made up of all edges connecting pairs of positive vertices. Both are trees.)

1.4. Towards an upper bound on distortion. It seems likely that $\operatorname{Dist}_{\Lambda_{k}}^{\Gamma_{k}} \simeq A_{k}$, but we do not offer a proof that $\operatorname{Dist}_{\Lambda_{k}}^{\Gamma_{k}} \preceq A_{k}$. The proof that $\operatorname{Dist}_{H_{k}}^{G_{k}} \preceq A_{k}$ in [6] may guide a proof that $\operatorname{Dist}_{\Lambda_{k}}^{\Gamma_{k}} \preceq A_{k}$, but that proof is technical and how to carry it over to Dist $\Gamma_{\Lambda_{k}}^{\Gamma_{k}}$ is not readily apparent. We are content to present here just the lower bound, which we believe is the more significant.

1.5. Height and quasiconvexity. A finitely generated subgroup $H$ of a finitely generated group $G$ is quasiconvex when $\operatorname{Dist}_{H}^{G}(n) \leq C n$ for some constant $C$. An infinite subgroup $H$ of a group $G$ has infinite height when, for all $n$, there exist $g_{1}$, $\ldots, g_{n}$ such that $\bigcap_{i=1}^{n} g_{i}^{-1} H g_{i}$ is infinite and $H g_{i} \neq H g_{j}$ for all $i \neq j$.

As $\Lambda_{k} \leq \Gamma_{k}$, for $k \geq 2$, are new examples of non-quasiconvex finitely presented subgroups of hyperbolic groups, they are test cases for the question attributed to Swarup in [13]: if a finitely presented subgroup $H$ of a hyperbolic group $G$ has finite height, is $H$ quasiconvex in $G$ ? (We thank Ilya Kapovich for drawing our attention to this.) 
Our $\Lambda_{k} \leq \Gamma_{k}$ do not resolve Swarup's question as they have infinite height for all $k \geq 1$. We explain this using the notation of Section 4. It follows from Proposition 4.8 that $t^{i} \in \Lambda_{k}$ if and only if $i=0$. So $\Lambda_{k} t^{i} \neq \Lambda_{k} t^{j}$ for all $i \neq j$. And $\bigcap_{i=1}^{\infty} t^{-i} \Lambda_{k} t^{i}$ is infinite since the rank $l$ free group $\left\langle b_{1}, \ldots, b_{l}\right\rangle$ is a subgroup of $t^{-i} \Lambda_{k} t^{i}$ for all $i$.

Acknowledgement. We thank an anonymous referee for a careful reading.

\section{Our examples}

2.1. A CAT(0) presentation for $\boldsymbol{\Gamma}_{\boldsymbol{k}}$. This presentation $P_{k}$ is well suited to establishing hyperbolicity (see Section 3 ):

$$
\begin{array}{ll}
\text { generators: } & \alpha_{1}, \ldots, \alpha_{k}, \beta_{1}, \ldots, \beta_{8}, \gamma_{1}, \ldots, \gamma_{8}, \sigma, \tau, \\
& \alpha_{i}^{-1} \tau \alpha_{i}=\alpha_{i-1}(1<i \leq k), \\
\text { relations: } \quad \beta_{i}^{-1} \tau \beta_{i}=\beta_{i+1}(1 \leq i \leq 7), \quad \beta_{8} \tau \beta_{8}^{-1}=\beta_{1}, \\
\gamma_{i}^{-1} \sigma \gamma_{i}=\gamma_{i+1}(1 \leq i \leq 7), \quad \gamma_{8} \sigma \gamma_{8}^{-1}=\gamma_{1}, \\
\gamma_{3} \beta_{5}=\beta_{3} \gamma_{5}, \alpha_{1} \gamma_{1} \tau=\tau \gamma_{7} \alpha_{1} .
\end{array}
$$

It is convenient to encode $P_{k}$ as shown in Figure 1 (which displays the case $k=6$ ). Each edge in the three labelled oriented trees (LOTs, see [10]) encodes a commutator relation, an edge labelled $y$ from a vertex labelled $x$ to a vertex labelled $z$ corresponds to a relation $y^{-1} x y=z$. The square and hexagonal 2-cells represent the remaining two relations, $\gamma_{3} \beta_{5}=\beta_{3} \gamma_{5}$ and $\alpha_{1} \gamma_{1} \tau=\tau \gamma_{7} \alpha_{1}$.

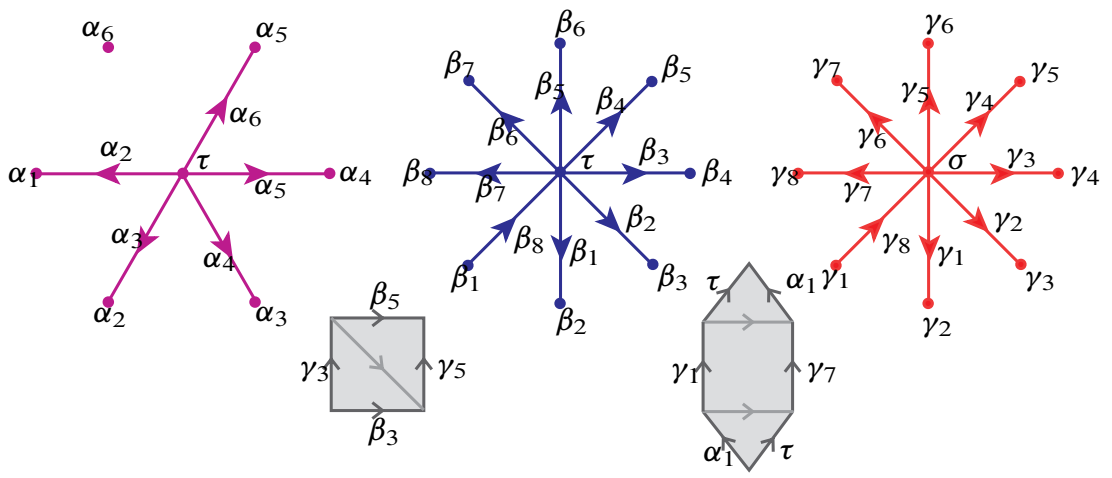

Figure 1. The defining relations of the presentation $P_{6}$ for $\Gamma_{6}$ displayed as three LOTs and two 2-cells.

If one removes the $\alpha_{i}$ and all the relations in which they appear from $P_{k}$, then one essentially gets groups studied by Mecham and Muckerjee in [11]. These, in turn, are built from two copies of groups studied by Barnard and the first author in [2]. 
2.2. A presentation of $\boldsymbol{\Gamma}_{\boldsymbol{k}}$ as a free-by-cyclic group. This presentation $Q_{k}$ has

generators: $\quad a_{0}, \ldots, a_{k}, b_{1}, \ldots, b_{8}, c_{1}, \ldots, c_{8}, d, t$,

$$
\text { relations: } \quad \begin{aligned}
t^{-1} a_{i} t & =\theta\left(a_{i}\right), \quad 0 \leq i \leq k, \\
t^{-1} b_{i} t & =\phi\left(b_{i}\right), \quad 1 \leq i \leq 8, \\
t^{-1} c_{i} t & =d \psi\left(c_{5}\right) \psi\left(c_{i}\right) \psi\left(c_{5}\right)^{-1} d^{-1}, \quad 1 \leq i \leq 8, \\
t^{-1} d t & =\phi^{2}\left(b_{5}\right)^{-1} d \psi\left(c_{5} c_{3}^{-1}\right) \phi\left(b_{3}\right),
\end{aligned}
$$

where $\theta, \phi$ and $\psi$ are defined by

$$
\begin{aligned}
\theta\left(a_{i}\right) & = \begin{cases}u a_{1} v, & i=0, \\
a_{0}, & i=1, \\
a_{i} a_{i-1}, & 1<i \leq k,\end{cases} \\
\phi\left(b_{i}\right) & =\left(b_{i} \ldots b_{7}\right) b_{1}^{-1} b_{8} \quad(1 \leq i \leq 8), \\
\psi\left(c_{i}\right) & =\left(c_{i} \ldots c_{8}\right) c_{1}^{-1} c_{8} \quad(1 \leq i \leq 8),
\end{aligned}
$$

and

$$
u=t^{-k} c_{7}^{-1} t d \psi\left(c_{5}\right) t^{k-1}, \quad v=t^{-(k-1)} \psi\left(c_{5}\right)^{-1} d^{-1} t^{-1} c_{1} t^{k}
$$

Lemma 2.1. $Q_{k}$ presents a free-by-cyclic group

$$
F\left(a_{0}, a_{1}, \ldots, a_{k}, b_{1}, \ldots, b_{8}, c_{1}, \ldots, c_{8}, d\right) \rtimes \mathbb{Z}
$$

where the $\mathbb{Z}$-factor is $\langle t\rangle$ and $t$ acts as an automorphism.

Proof. First note:

(i) $u$ and $v$ represent elements of the subgroup $\left\langle b_{1}, \ldots, b_{8}, c_{1}, \ldots, c_{8}, d\right\rangle$, and

(ii) $\phi$ and $\psi$ define automorphisms of $F\left(b_{1}, \ldots, b_{8}\right)$ and $F\left(c_{1}, \ldots, c_{8}\right)$, respectively, as would $\theta$ for $F\left(a_{0}, \ldots, a_{k}\right)$ were $\theta\left(a_{0}\right)$ equal to $a_{1}$ rather than $u a_{1} v$.

The action of $t$ by conjugation on

$$
F\left(a_{0}, a_{1}, \ldots, a_{k}, b_{1}, \ldots, b_{8}, c_{1}, \ldots, c_{8}, d\right)
$$

apparent in the presentation $Q_{k}$ is an automorphism because, as we will explain, the 
following is a sequence of free bases:

$$
\begin{aligned}
& \left(a_{0}, a_{1}, \ldots, a_{k}, b_{1}, \ldots, b_{8}, c_{1}, \ldots, c_{8}, d\right) \\
& \stackrel{(1)}{\longrightarrow}\left(a_{1}, t^{-1} a_{1} t, \ldots, t^{-1} a_{k} t, b_{1}, \ldots, b_{8}, c_{1}, \ldots, c_{8}, d\right) \\
& \stackrel{(2)}{\longrightarrow}\left(t^{-1} a_{0} t, t^{-1} a_{1} t, \ldots, t^{-1} a_{k} t, b_{1}, \ldots, b_{8}, c_{1}, \ldots, c_{8}, d\right) \\
& \stackrel{(3)}{\longrightarrow}\left(t^{-1} a_{0} t, t^{-1} a_{1} t, \ldots, t^{-1} a_{k} t, t^{-1} b_{1} t, \ldots, t^{-1} b_{8} t, \psi\left(c_{1}\right), \ldots, \psi\left(c_{8}\right), d\right) \\
& \stackrel{(4)}{\longrightarrow}\left(t^{-1} a_{0} t, t^{-1} a_{1} t, \ldots, t^{-1} a_{k} t, t^{-1} b_{1} t, \ldots, t^{-1} b_{8} t, \psi\left(c_{1}\right), \ldots\right. \\
& \stackrel{(5)}{\longrightarrow}\left(t^{-1} a_{0} t, t^{-1} a_{1} t, \ldots, t^{-1} a_{k} t, t^{-1} b_{1} t, \ldots, t^{-1} b_{8} t, t^{-1} c_{1} t, \ldots\right. \\
& \stackrel{(6)}{\longrightarrow}\left(t^{-1} a_{0} t, t^{-1} a_{1} t, \ldots, t^{-1} c_{8} t, d \psi\left(c_{5} c_{3}^{-1}\right)\right)
\end{aligned}
$$

This is because (1) $a_{1}, t^{-1} a_{1} t, \ldots, t^{-1} a_{k} t$ is a free basis for $F\left(a_{0}, \ldots, a_{k}\right)$ as per (ii) above; (2) $t^{-1} a_{0} t=u a_{1} v$, which is equivalent via transvections to $a_{1}$ by (i);

(3) follows from (ii); (4) is via transvections; (5) conjugation by $\psi\left(c_{5}\right)^{-1} d^{-1}=$ $\psi\left(c_{5}\right)^{-1} \psi\left(c_{5} c_{3}^{-1}\right) \psi\left(c_{5} c_{3}^{-1}\right)^{-1} d^{-1}$ is first conjugation by $\psi\left(c_{5}\right)^{-1} \psi\left(c_{5} c_{3}^{-1}\right)$, which is an automorphism of $F\left(c_{1}, \ldots, c_{8}\right)$, and then by $\psi\left(c_{5} c_{3}^{-1}\right)^{-1} d^{-1}$; and (6) is via transvections as $t^{-1} b_{1} t, \ldots, t^{-1} b_{8} t$ are a free basis for $F\left(b_{1}, \ldots, b_{8}\right)$ and $\phi^{2}\left(b_{5}\right)^{-1}, \phi\left(b^{3}\right) \in F\left(b_{1}, \ldots, b_{8}\right)$.

The subgroup $\Lambda_{k}$ of Theorem 1.1 will be

$$
\left\langle a_{0} t, \ldots, a_{k} t, b_{1}, \ldots, b_{8}, c_{1}, \ldots, c_{8}, d\right\rangle \text {. }
$$

2.3. The equivalence of the presentations. We will prove:

Proposition 2.2. $P_{k}$ and $Q_{k}$ present the same groups.

As a first step we establish:

Lemma 2.3. Mapping $\tau \mapsto t^{-1}$ and $\beta_{i} \mapsto t^{-1} b_{i}$ for $1 \leq i \leq 8$ defines an isomorphism

$$
\begin{aligned}
& \left\langle\beta_{1}, \ldots, \beta_{8}, \tau \mid \beta_{i}^{-1} \tau \beta_{i}=\beta_{i+1}(1 \leq i \leq 7), \beta_{8} \tau \beta_{8}^{-1}=\beta_{1}\right\rangle \\
& \rightarrow F\left(b_{1}, \ldots, b_{8}\right) \rtimes_{\phi} \mathbb{Z}=\left\langle b_{1}, \ldots, b_{8}, t \mid t^{-1} b_{i} t=\phi\left(b_{i}\right) \quad(1 \leq i \leq 8)\right\rangle .
\end{aligned}
$$

Proof. The given map translates the relations $\beta_{i}^{-1} \tau \beta_{i}=\beta_{i+1}(1 \leq i \leq 7)$ and $\beta_{8} \tau \beta_{8}^{-1}=\beta_{1}$ to the family

$$
t^{-1} b_{i} t=b_{i} t^{-1} b_{i+1} t(1 \leq i \leq 7), \quad t^{-1} b_{8} t=b_{1}^{-1} b_{8},
$$

which is equivalent to $t^{-1} b_{i} t=\phi\left(b_{i}\right)(1 \leq i \leq 8)$. 
Let $P_{k}^{\prime}$ and $Q_{k}^{\prime}$ be the presentation obtained from $P_{k}$ and $Q_{k}$ by removing all the generators $\alpha_{i}$ and $a_{i}$, respectively, and all the relations in which they occur.

Lemma 2.4. The groups presented by $P_{k}^{\prime}$ and $Q_{k}^{\prime}$ are isomorphic via

$$
\begin{array}{ccc}
\tau \mapsto t^{-1}, & \beta_{i} \mapsto t^{-1} b_{i} & (1 \leq i \leq 8), \\
\sigma \mapsto s^{-1}, & \gamma_{i} \mapsto s^{-1} c_{i} & (1 \leq i \leq 8),
\end{array}
$$

where $s=t d \psi\left(c_{5}\right)$.

Proof. As per Lemma 2.3, translate $\beta_{1}, \ldots, \beta_{8}, \tau$ and associated relations to $b_{1}, \ldots$, $b_{8}, t$ and $\gamma_{1}, \ldots, \gamma_{8}, \sigma$ and associated relations to $c_{1}, \ldots, c_{8}, s$.

The given map converts the relation $\gamma_{3} \beta_{5}=\beta_{3} \gamma_{5}$ to

$$
s^{-1} c_{3} t^{-1} b_{5}=t^{-1} b_{3} s^{-1} c_{5} .
$$

This rearranges as

$$
t^{-1} b_{5} c^{-5} s b_{3}^{-1} t=c_{3}^{-1} s
$$

and then as

$$
\left(t^{-1} b_{5} t\right) t^{-1} s\left(s^{-1} c_{5}^{-1} s\right) t\left(t^{-1} b_{3}^{-1} t\right)=s\left(s^{-1} c_{3}^{-1} s\right),
$$

which is equivalent to

$$
\phi\left(b_{5}\right) t^{-1} s \psi\left(c_{5}\right)^{-1} t \phi\left(b_{3}\right)^{-1}=s \psi\left(c_{3}\right)^{-1},
$$

and so to

$$
t^{-1} s \psi\left(c_{5}\right)^{-1} t=t^{-1} \phi\left(b_{5}\right)^{-1} s \psi\left(c_{3}\right)^{-1} \phi\left(b_{3}\right) .
$$

So, as $s=t d \psi\left(c_{5}\right)$,

$$
t^{-1} d t=t^{-1} \phi\left(b_{5}\right)^{-1} t d \psi\left(c_{5}\right) \psi\left(c_{3}\right)^{-1} \phi\left(b_{3}\right),
$$

which gives

$$
t^{-1} d t=\phi^{2}\left(b_{5}\right)^{-1} d \psi\left(c_{5} c_{3}^{-1}\right) \phi\left(b_{3}\right)
$$

as per $Q_{k}^{\prime}$. Next, as $s=t d \psi\left(c_{5}\right)$, the relation $s^{-1} c_{i} s=\psi\left(c_{i}\right)$ is equivalent to

$$
t^{-1} c_{i} t=d \psi\left(c_{5}\right) \psi\left(c_{i}\right) \psi\left(c_{5}\right)^{-1} d^{-1}
$$

as per $Q_{k}^{\prime}$.

Inductively define words $u_{i}$ and $v_{i}$ for $i \geq 0$ by

$$
\begin{aligned}
& u_{0}=\alpha_{k}, \quad u_{i+1}=u_{i}^{-1} t^{-1} u_{i} \quad(i \geq 0), \\
& v_{0}=a_{k}, \quad v_{i+1}=v_{i}^{-1} t^{-1} v_{i} t \quad(i \geq 0) .
\end{aligned}
$$

The following observation from [6] can be proved by inducting on $i$. 
Lemma 2.5. On substituting an $a_{k}$ for each $\alpha_{k}$ in $u_{i}$, the words $u_{i}$ and $t^{i-1} v_{i} t^{-i}$ become freely equal for all $i \geq 1$.

Proof of Proposition 2.2. By Lemma 2.4 there is a sequence of Tietze moves carrying the subpresentation $P_{k}^{\prime}$ of $P_{k}$ to $Q_{k}^{\prime}$ and the remaining relations (those involving the $\left.\alpha_{i}\right)$ to

$$
\alpha_{1} s^{-1} c_{1} t^{-1}=t^{-1} s^{-1} c_{7} \alpha_{1}, \quad \alpha_{i}^{-1} t^{-1} \alpha_{i}=\alpha_{i-1}, \quad 1<i \leq k .
$$

A sequence of Tietze moves eliminating $\alpha_{1}, \ldots, \alpha_{k-1}$ transforms this family to the single relation

$$
u_{k-1} s^{-1} c_{1} t^{-1}=t^{-1} s^{-1} c_{7} u_{k-1} .
$$

Now substitute an $a_{k}$ for each $\alpha_{k}$. Then, by Lemma 2.5, this relation is equivalent to

$$
\left(t^{k-2} v_{k-1} t^{-(k-1)}\right) s^{-1} c_{1} t^{-1}=t^{-1} s^{-1} c_{7}\left(t^{k-2} v_{k-1} t^{-(k-1)}\right),
$$

which becomes

$$
t^{-1} v_{k-1} t=\left(t^{-(k-1)} c_{7}^{-1} s t^{k-1}\right) v_{k-1}\left(t^{-(k-1)} s^{-1} c_{1} t^{k-1}\right)
$$

on conjugating by $t^{k-1}$ and rearranging. A sequence of Tietze moves introducing $a_{k-1}, \ldots, a_{1}$ expands this to the family

$t^{-1} a_{1} t=t^{-(k-1)} c_{7}^{-1} s t^{k-1} a_{1} t^{-(k-1)} s^{-1} c_{1} t^{k-1}, \quad t^{-1} a_{i} t=a_{i} a_{i-1}, \quad 1<i \leq k$.

The first of these relations becomes $t^{-1} a_{1} t=a_{0}$ when we introduce $a_{0}$ together with the new relation

$$
a_{0}=t^{-(k-1)} c_{7}^{-1} s t^{k-1} a_{1} t^{-(k-1)} s^{-1} c_{1} t^{k-1},
$$

which becomes $t^{-1} a_{0} t=u a_{1} v$ on conjugating by $t$ and eliminating the $s$ and $s^{-1}$ using $s=t d \psi\left(c_{5}\right)$.

\section{Hyperbolicity}

We establish hyperbolicity using techniques employed in [2] and [11].

Consider the presentation 2-complex $K_{k}$ for $P_{k}$ assembled from Euclidean unit squares associated to each of the defining relations with the single exception of $\alpha_{1} \gamma_{1} \tau=\tau \gamma_{7} \alpha_{1}$ for which we use a Euclidean hexagon made from one unit square and two equilateral triangles as shown in Figure 1.

The link in the case $k=6$ is shown in Figure 2. All edges have length $\pi / 2$ apart from the edges $\tau^{+}-\alpha_{1}^{+}$and $\tau^{-}-\alpha_{1}^{-}$(shown in grey), which have length $\pi / 3$, and the edges from $\gamma_{1}^{+}-\tau^{-}, \gamma_{7}^{+}-\alpha_{1}^{-}, \alpha_{1}^{+}-\gamma_{1}^{-}$, and $\tau^{+}-\gamma_{7}^{+}$(shown in green), which have length $5 \pi / 6$. Inspecting the link we see that any simple loop in the graph has length at 


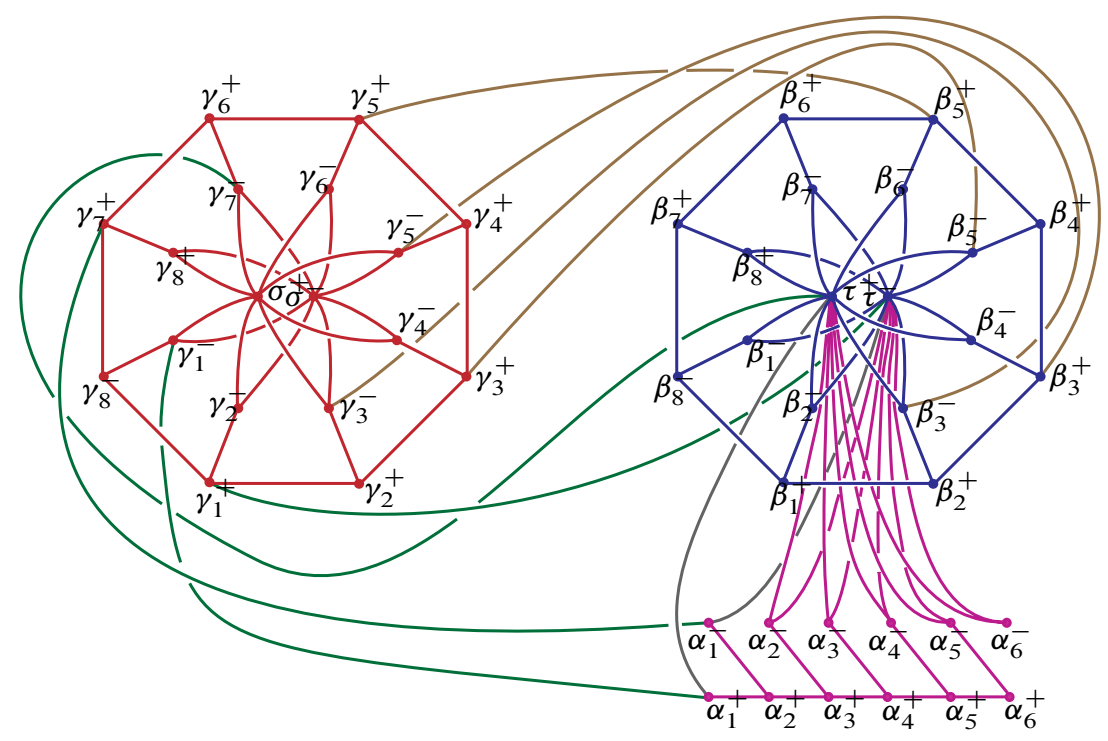

Figure 2. The link of the vertex in the presentation 2-complex associated to the presentation $P_{6}$ for $\Gamma_{6}$ given in Section 2 . The two grey edges have length $\pi / 3$, the four green edges have length $5 \pi / 6$, and all other edges have length $\pi / 2$.

least $2 \pi$ (separately considering the cases of monochrome and multicoloured simple loops in Figure 1 helps to check this) - that is, for all $k \geq 1$ the link is large. So $K_{k}$ satisfies the link condition (see [5]) and its universal cover $\widetilde{K_{k}}$ is therefore a CAT(0) space.

To establish that $\Gamma_{k}$ is hyperbolic we will show that $\widetilde{K_{k}}$ contains no subspace isometric to $\mathbb{E}^{2}$ and then appeal to the Flat Plane Theorem of [4], [8]. The link of a vertex in any isometric copy of $\mathbb{E}^{2}$ in $\widetilde{K_{k}}$ would appear as a simple loop of length $2 \pi$ in the link. But inspecting the link, we find that no edges of length $\pi / 3$ or $5 \pi / 6$ (the grey and green edges) occur in a simple loop of length $2 \pi$. Next one can check the edges $\gamma_{3}^{+}-\beta_{5}^{-}, \gamma_{3}^{-}-\beta_{3}^{-}, \gamma_{5}^{-}-\beta_{3}^{+}$and $\gamma_{5}^{+}-\beta_{5}^{+}$(the brown edges in the figure) do not occur in a simple loop of length $2 \pi$. Then it becomes evident that edges occurring in simple loops of length $2 \pi$ are precisely the edges

$$
\begin{aligned}
& \tau^{+}-\alpha_{i}^{-}, \quad \tau^{-}-\alpha_{i}^{-} \quad(2 \leq i \leq k), \\
& \tau^{+}-\beta_{i}^{-}, \quad \tau^{-}-\beta_{i}^{-} \quad(1 \leq i \leq 7), \quad \tau^{+}-\beta_{8}^{+}, \quad \tau^{-}-\beta_{8}^{+}, \\
& \sigma^{+}-\gamma_{i}^{-}, \quad \sigma^{-}-\gamma_{i}^{-} \quad(1 \leq i \leq 7), \quad \sigma^{+}-\gamma_{8}^{+}, \quad \sigma^{-}-\gamma_{8}^{+} \text {. }
\end{aligned}
$$

So every corner of every 2-cell in an isometrically embedded copy of $\mathbb{E}^{2}$ must give rise to one of the edges in this list. But, looking at the defining relations, we see that no 2-cell in $\widetilde{K_{k}}$ has this property. Therefore there are no such $\mathbb{E}^{2}$, and so $\Gamma_{k}$ is hyperbolic. 


\section{Freeness and distortion}

4.1. A family of free-by-cyclic groups. Fix an integer $l \geq 1$, words $u$ and $v$ on $b_{1}, \ldots, b_{l}$, and an automorphism $\phi$ of $F\left(b_{1}, \ldots, b_{l}\right)$. Then, for $k \geq 1$, define

$$
\Psi_{k}:=F\left(a_{0}, \ldots, a_{k}, b_{1}, \ldots, b_{l}\right) \rtimes_{\theta} \mathbb{Z},
$$

where $\theta$ is the automorphism of $F\left(a_{0}, \ldots, a_{k}, b_{1}, \ldots, b_{l}\right)$ the restriction of which to $F\left(b_{1}, \ldots, b_{l}\right)$ is $\phi$ and

$$
\theta\left(a_{i}\right)= \begin{cases}u a_{1} v, & i=0, \\ a_{0}, & i=1, \\ a_{i} a_{i-1}, & 1<i \leq k\end{cases}
$$

Let $t$ denote a generator of the $\mathbb{Z}$-factor, so $t^{-1} a_{i} t=\theta\left(a_{i}\right)$ and $t^{-1} b_{j} t=\theta\left(b_{j}\right)$ for all $i$ and $j$.

The presentation $Q_{k}$ in Section 2.2 shows $\Gamma_{k}$ is an example of such a $\Psi_{k}$.

Our aim in the remainder of this section is to establish:

Proposition 4.1. The subgroup

$$
\Lambda_{k}:=\left\langle a_{0} t, \ldots, a_{k} t, b_{1}, \ldots, b_{l}\right\rangle
$$

of $\Psi_{k}$ is free of rank $k+l+1$ and $\operatorname{Dist}_{\Lambda_{k}}^{\Psi_{k}} \succeq A_{k}$.

4.2. Towards a lower bound on distortion. In what follows, when, for a word $u=u\left(a_{0}, \ldots, a_{k}, b_{1}, \ldots, b_{l}\right)$, we refer to $\theta^{r}(u)$, we mean the freely reduced word that equals $\theta^{r}(u)$ in $F\left(a_{0}, \ldots, a_{k}, b_{1}, \ldots, b_{l}\right)$.

The extreme distortion in the hydra groups of [6] stemmed from the battle between Hercules and the hydra that we described in Section 1. When studying $\Psi_{k}$ we will need the following more elaborate version of that battle. A hydra is now a word on

$$
a_{0}, a_{1}, \ldots, a_{k}, b_{1}, \ldots, b_{l}
$$

in which the $a_{i}$ only appear with positive exponents. As before, Hercules fights a hydra by removing the first letter. But in this version, the hydra only regenerates after an $a_{i}$ is removed, and that regeneration is: each remaining $a_{i}$ and $b_{j}^{ \pm 1}$ becomes $\theta\left(a_{i}\right)$ and $\theta\left(b_{j}^{ \pm 1}\right)$, respectively. Again, we consider Hercules victorious if, on sufficient repetition, the hydra is reduced to the empty word.

Reprising the example from Section 1, Hercules defeats $a_{2}^{3}$ as follows:

$$
\begin{gathered}
a_{2}^{3} \rightarrow\left(a_{2} a_{1}\right)^{2} \rightarrow a_{0} a_{2} a_{1} a_{0} \rightarrow a_{2} a_{1} a_{0} u a_{1} v \rightarrow a_{0} u a_{1} v \theta(u) a_{0} \theta(v) \\
\rightarrow a_{0} \theta(v) \theta^{2}(u) u a_{1} v \theta^{2}(v) \rightarrow a_{0} \theta(v) \theta^{3}(v) \rightarrow \varepsilon .
\end{gathered}
$$


Here the steps in which Hercules removes a $b_{j}$ are not shown; the arrows indicate the progression from when an $a_{i}$ is about to be removed to when an $a_{i}$ next appears at the front of the word or the hydra becomes the empty word.

The salient point is that $a_{0}$ and the $b_{j}$ play no essential role in this battle; if we removed all $b_{j}$ and replaced all $a_{0}$ by $a_{1}$, we would have a battle of the original form. Thus we have the following lemma. (Recall that $\mathscr{H}(u)$ denotes the duration of the battle (of the original type from Section 1 and [6]) against the hydra $u$.)

Lemma 4.2. Hercules wins against all hydra $w$ and, in the battle, the number of times he removes an $a_{i}$ equals $\mathscr{H}(\bar{w})$ where $\bar{w}$ is the word obtained from $w$ by removing all $b_{j}^{ \pm 1}$ and replacing all $a_{0}$ by $a_{1}$.

Consideration of the original battle between Hercules and the hydra led to the result that, for all $k, n \geq 1$, there is a positive word $u_{k, n}=u_{k, n}\left(a_{1} t, \ldots, a_{k} t\right)$ of length $\mathscr{H}_{k}(n)$ that equals $a_{k}^{n} t^{\mathscr{H}_{k}(n)}$ in $G_{k}$. (This is Lemma 5.1 in [6].) The reason is that the pairing off of a $t$ with an initial $a_{i}$ in a positive word on $a_{1}, \ldots, a_{k}$ corresponds to a decapitation, and the conjugation by $t$ that moves that $t$ into place from the righthand end causes regeneration for the remainder of the word. For example $\mathscr{H}_{2}(3)=7$ and

$$
\begin{aligned}
a_{2}^{3} t^{7} & =\left(a_{2} t\right) t^{-1} a_{2}^{2} t t^{6} \\
& =\left(a_{2} t\right)\left(a_{2} a_{1}\right)^{2} t^{6} \\
& =\left(a_{2} t\right)\left(a_{2} t\right) t^{-1} a_{1} a_{2} a_{1} t t^{5} \\
& \vdots \\
& =\left(a_{2} t\right)\left(a_{2} t\right)\left(a_{1} t\right)\left(a_{2} t\right)\left(a_{1} t\right)\left(a_{1} t\right)\left(a_{1} t\right) \\
& =u_{2,3} .
\end{aligned}
$$

In the corresponding calculation for $\Psi_{k}$, only the $a_{i}$ get paired with $t$, and on each of the $\mathscr{H}_{k}(n)$ times that happens, the subsequent conjugation by $t$ can increase length by a factor $C$ which depends only on $\phi, \ell(u)$ and $\ell(v)$. So:

Lemma 4.3. There exists $C>0$ such that for all $k, n \geq 1$ there is a word $\hat{u}_{k, n}=$ $\hat{u}_{k, n}\left(a_{0} t, \ldots, a_{k} t, b_{1}, \ldots, b_{l}\right)$ that equals $a_{k}^{n} t^{\mathscr{H}_{k}(n)}$ in $\Psi_{k}$ and has the properties that

$$
\mathscr{H}_{k}(n) \leq \ell\left(\hat{u}_{k, n}\right) \leq C^{\mathscr{H}_{k}(n)} n
$$

and all the $\left(a_{i} t\right)$ it contains have positive exponents.

This and our next two lemmas will be components of a calculation that will yield Proposition 4.6 (the analogue of Proposition 5.2 in [6]), which will be the key to establishing a lower bound on the distortion of $\Lambda_{k}$ in $\Psi_{k}$.

A simple calculation yields: 
Lemma 4.4. $t^{-m} a_{1} t^{m+1}=\tau_{m}$ in $\Psi_{k}$ for all $m \geq 1$ where

$$
\tau_{m}:= \begin{cases}a_{0} t & \text { for } m=1, \\ \phi^{m-2}(u) \ldots \phi^{2}(u) u\left(a_{1} t\right) \phi(v) \phi^{3}(v) \ldots \phi^{m-1}(v) & \text { for even } m \geq 2, \\ \phi^{m-2}(u) \ldots \phi^{3}(u) \phi(u)\left(a_{0} t\right) \phi^{2}(v) \phi^{4}(v) \ldots \phi^{m-1}(v) & \text { for odd } m>2 .\end{cases}
$$

This combines with

$$
t^{-1}\left(a_{1} t^{-1}\right)^{n}=\left(t^{-1} a_{1} t^{2}\right)\left(t^{-3} a_{1} t^{4}\right)\left(t^{-5} a_{1} t^{6}\right) \ldots\left(t^{1-2 n} a_{1} t^{2 n}\right) t^{-2 n-1}
$$

to give:

Lemma 4.5. There is a constant $C>0$, depending only on $\phi, \ell(u)$ and $\ell(v)$, such that for all $n \geq 0$ there is a word $v_{n}=v_{n}\left(a_{0} t, b_{1}, \ldots, b_{l}\right)$ such that $t^{-1}\left(a_{1} t^{-1}\right)^{n}=$ $v_{n} t^{-2 n-1}$ in $\bar{\Psi}_{k}$, the number of $\left(a_{0} t\right)$ contained in $v_{n}$ is $n$ and all have positive exponent, and $n \leq \ell\left(v_{n}\right) \leq C^{n}$.

Proposition 4.6. For all $k \geq 2$ and $n \geq 1$, there is a reduced word of length at least $2 \mathscr{H}_{k}(n)+3$ on $a_{0} t, a_{1} t, \ldots, a_{k} t, b_{1}, \ldots, b_{l}$ that equals $a_{k}^{n} a_{2} t a_{1} a_{2}^{-1} a_{k}^{-n}$ in $\Psi_{k}$.

Proof. After rewriting the relation $t^{-1} a_{2} t=a_{2} a_{1}$ as $a_{2}^{-1} t a_{2}=t a_{1}^{-1}$, we see $a_{2}^{-1} t^{\mathscr{H}_{k}(n)} a_{2}=\left(t a_{1}^{-1}\right)^{\mathscr{H}_{k}(n)}$. So

$$
a_{k}^{n} a_{2}=\hat{u}_{k, n} a_{2}\left(t a_{1}^{-1}\right)^{-\mathscr{H}_{k}(n)}
$$

for $\hat{u}_{k, n}$ as in Lemma 4.3. This gives the first of the equalities

$$
\begin{aligned}
a_{k}^{n} a_{2} t a_{1} a_{2}^{-1} a_{k}^{-n} & =\hat{u}_{k, n} a_{2}\left(t a_{1}^{-1}\right)^{-\mathscr{H}_{k}(n)} t a_{1}\left(t a_{1}^{-1}\right)^{\mathscr{H}_{k}(n)} a_{2}^{-1} \hat{u}_{k, n}^{-1} \\
& =\hat{u}_{k, n}\left(a_{2} t\right) t^{-1}\left(t a_{1}^{-1}\right)^{-\mathscr{H}_{k}(n)} t a_{1}\left(t a_{1}^{-1}\right)^{\mathscr{H}_{k}(n)} t\left(a_{2} t\right)^{-1} \hat{u}_{k, n}^{-1} \\
& =\hat{u}_{k, n}\left(a_{2} t\right) v_{\mathscr{H}_{k}(n)} t^{-2 \mathscr{H}_{k}(n)-1} t a_{1} t^{2 \mathscr{H}_{k}(n)+1} v_{\mathscr{H}_{k}(n)}^{-1}\left(a_{2} t\right)^{-1} \hat{u}_{k, n}^{-1} \\
& =\hat{u}_{k, n}\left(a_{2} t\right) v_{\mathscr{H}_{k}(n)} \tau_{2 \mathscr{H}_{k}(n)} v_{\mathscr{H}_{k}(n)}^{-1}\left(a_{2} t\right)^{-1} \hat{u}_{k, n}^{-1} .
\end{aligned}
$$

The second is a free equality and the third and fourth are applications of Lemmas 4.5 and 4.4 , respectively.

This calculation arrives at a word on $a_{0} t, a_{1} t, \ldots, a_{k} t, b_{1}, \ldots, b_{l}$ that equals $a_{k}^{n} a_{2} t a_{1} a_{2}^{-1} a_{k}^{-n}$ in $\Psi_{k}$. This word may not be freely reduced, but if we delete all the $b_{j}^{ \pm 1}$ it contains, replace all $a_{0}^{ \pm 1}$ by $a_{1}^{ \pm 1}$, and then freely reduce (i.e., cancel away all $\left(a_{i} t\right)^{ \pm 1}\left(a_{i} t\right)^{\mp 1}$ subwords), we get $u_{k, n}\left(a_{2} t\right)\left(a_{1} t\right)\left(a_{2} t\right)^{-1} u_{k, n}^{-1}$, which has length $2 \mathscr{H}_{k}(n)+3$.

4.3. Freeness and rank. The result of this section is:

Proposition 4.7. The subgroup $\Lambda_{k}$ is free of rank $k+l+1$. 
It will be convenient to prove more. In the special case where $w$ represents the identity, the following proposition tells us that there are no non-trivial relations between $a_{0} t, \ldots, a_{k} t, b_{1}, \ldots, b_{l}$ and so establishes Proposition 4.7.

Proposition 4.8. If $w=w\left(a_{0} t, \ldots, a_{k} t, b_{1}, \ldots, b_{l}\right)$ represents an element of the subgroup $\langle t\rangle$ in $\Psi_{k}$, then $w$ freely equals the empty word.

We begin with an observation on how the groups $\Psi_{k}$ nest.

Lemma 4.9. For $1 \leq i \leq k$, the canonical homomorphism $\Psi_{i} \rightarrow \Psi_{k}$ is an inclusion.

Proof. The free-by-cyclic normal forms - a reduced word on $a_{0}, \ldots, a_{k}, b_{1}, \ldots, b_{l}$ times a power of $t$ - of an element of $\Psi_{i}$ and its image in $\Psi_{k}$ are the same.

We will prove Proposition 4.8 by induction, but first we give a corollary which will be useful in the induction step. We emphasise that when we say that $v\left(a_{0} t, \ldots, a_{k} t, b_{1}, \ldots, b_{l}\right)$ is freely reduced in the following we mean that there are no $\left(a_{i} t\right)^{ \pm 1}\left(a_{i} t\right)^{\mp 1}$ or $b_{j}^{ \pm 1} b_{j}^{\mp 1}$ subwords.

Corollary 4.10. Suppose that $v\left(a_{0} t, \ldots, a_{k} t, b_{1}, \ldots, b_{l}\right)$ is a freely reduced word equalling $\hat{v} t^{s}$ in $\Psi_{k}$, where $s \in \mathbb{Z}$ and $\hat{v}=\hat{v}\left(a_{0}, \ldots, a_{k}, b_{1}, \ldots, b_{l}\right)$ is a word in which all the $a_{i}$ that occur have positive exponents. Then all the $\left(a_{i} t\right)$ in $v$ have positive exponents.

Proof. When played out against $\hat{v}\left(a_{0}, \ldots, a_{k}, b_{1}, \ldots, b_{l}\right)$, the hydra battle described prior to Lemma 4.2 gives a word $v^{\prime}=v^{\prime}\left(a_{0} t, \ldots, a_{k} t, b_{1}, \ldots, b_{l}\right)$ and an integer $s^{\prime}$ such that $v^{\prime}=\hat{v} t^{s^{\prime}}$ in $\Psi_{k}$. Moreover, the exponents of all the $\left(a_{i} t\right)$ in $v^{\prime}$ are positive. Now $v^{-1} v^{\prime} \in\langle t\rangle$ since $\hat{v}=v t^{-s}=v^{\prime} t^{-s^{\prime}}$, and so $v$ and $v^{\prime}$ are freely equal by Proposition 4.8. Therefore the exponents of all the $\left(a_{i} t\right)$ in $v$ are positive.

Proof of Proposition 4.8. We induct on $k$. For the base case of $k=1$, notice that defining $\bar{a}_{0}:=a_{0} t$ and $\bar{a}_{1}:=a_{1} t$, we can transform the presentation

$$
\left.\left\langle a_{0}, a_{1}, b_{1}, \ldots, b_{l}, t\right| t^{-1} a_{0} t=u a_{1} v, t^{-1} a_{1} t=a_{0}, t^{-1} b_{j} t=\phi\left(b_{j}\right) \text { for all } j\right\rangle
$$

for $\Psi_{1}$ to

$\left\langle\bar{a}_{0}, \bar{a}_{1}, b_{1}, \ldots, b_{l}, t\right| t^{-1} \bar{a}_{0} t=u \bar{a}_{1} \phi(v), t^{-1} \bar{a}_{1} t=\bar{a}_{0}, t^{-1} b_{j} t=\phi\left(b_{j}\right)$ for all $\left.j\right\rangle$,

which is an alternative means of expressing $\Psi_{1}$ as a free-by-cyclic group from which the result is evident.

For the induction step, we consider a freely reduced word $w=w\left(a_{0} t, \ldots, a_{k} t\right.$, $\left.b_{1}, \ldots, b_{l}\right)$ representing an element of $\langle t\rangle$ in $\Psi_{k}$ where $k \geq 2$. If no $\left(a_{k} t\right)^{ \pm 1}$ are present in $w$ we can deduce from the induction hypothesis and Lemma 4.9 that $w$ 
freely reduces to the empty word. For the remainder of our proof we suppose there are $\left(a_{k} t\right)^{ \pm 1}$ present, and we seek a contradiction.

Consider shuffling the $t^{ \pm 1}$ to the start of $w$ using the defining relations - replacing each $a_{i}$ and $b_{j}$ passed by a $t^{ \pm 1}$ with $\theta^{ \pm 1}\left(a_{i}\right)$ and $\theta^{ \pm 1}\left(b_{j}\right)$, respectively. The result will be a power of $t$ times a word on $a_{0}, \ldots, a_{k}, b_{1}, \ldots, b_{l}$ which freely reduces to the empty word. Such is $\theta$, no $a_{k}$ are created or destroyed in this process of shuffling the $t^{ \pm 1}$. So there is some expression $w_{0}\left(a_{k} t\right)^{ \pm 1} u\left(a_{k} t\right)^{\mp 1} w_{1}$ for $w$ such that $u=u\left(a_{0} t, \ldots, a_{k-1} t, b_{1}, \ldots, b_{l}\right)$ and the $a_{k}^{ \pm 1}$ and $a_{k}^{\mp 1}$ in the $\left(a_{k} t\right)^{ \pm 1}$ and $\left(a_{k} t\right)^{\mp 1}$ buttressing $u$ cancel after the shuffling and free reduction.

We will address first the case $w=w_{0}\left(a_{k} t\right)^{-1} u\left(a_{k} t\right) w_{1}$. Break down the shuffling process by first shuffling the $t^{ \pm 1}$ out of $w_{0}, u$ and $w_{1}$, and then carrying the resulting powers to the front of the word:

$$
\begin{aligned}
& w=w_{0}\left(a_{k} t\right)^{-1} u\left(a_{k} t\right) w_{1} \rightarrow t^{r_{0}} \hat{w}_{0}\left(a_{k} t\right)^{-1} t^{r} \hat{u}\left(a_{k} t\right) t^{r_{1}} \widehat{w}_{1} \\
& \rightarrow t^{r_{0}+r+r_{1}} \theta^{r+r_{1}}\left(\hat{w}_{0}\right) \theta^{r+r_{1}+1}\left(a_{k}^{-1}\right) \theta^{r_{1}+1}(\hat{u}) \theta^{r_{1}+1}\left(a_{k}\right) \hat{w}_{1},
\end{aligned}
$$

where $r_{0}, r, r_{1} \in \mathbb{Z}$ and

$$
\begin{aligned}
\widehat{w}_{0} & =\widehat{w}_{0}\left(a_{0}, \ldots, a_{k}, b_{1}, \ldots, b_{l}\right), \\
\hat{u} & =\hat{u}\left(a_{0}, \ldots, a_{k-1}, b_{1}, \ldots, b_{l}\right), \\
\widehat{w}_{1} & =\widehat{w}_{1}\left(a_{0}, \ldots, a_{k}, b_{1}, \ldots, b_{l}\right)
\end{aligned}
$$

are words such that $t^{r_{0}} \widehat{w}_{0}=w_{0}, t^{r} \hat{u}=u$ and $t^{r_{1}} \widehat{w}_{1}=w_{1}$ in $\Psi_{k}$. When we expand $\theta^{r+r_{1}}\left(a_{k}^{-1}\right)$ and $\theta^{r_{1}+1}\left(a_{k}\right)$ as words on $a_{0}, \ldots, a_{k}$, the former ends with an $a_{k}^{-1}$ which must cancel with the $a_{k}$ at the start of the latter. So $\theta^{r_{1}+1}(\hat{u})$, and therefore $\hat{u}$, freely equal the empty word. So $u$ represents an element of $\langle t\rangle$ and, by induction hypothesis, freely reduces to the empty word, contrary to the initial assumption that $w\left(a_{0} t, \ldots a_{k} t, b_{1}, \ldots, b_{l}\right)$ is reduced.

In the case $w=w_{0}\left(a_{k} t\right) u\left(a_{k} t\right)^{-1} w_{1}$, the shuffling process is

$$
\begin{aligned}
w=w_{0}\left(a_{k} t\right) u\left(a_{k} t\right)^{-1} w_{1} \rightarrow t^{r_{0}} \widehat{w}_{0}\left(a_{k} t\right) t^{r} \hat{u}\left(a_{k} t\right)^{-1} t^{r_{1}} \widehat{w}_{1} \\
\rightarrow t^{r_{0}+r+r_{1}} \theta^{r+r_{1}}\left(\hat{w}_{0}\right) \theta^{r+r_{1}}\left(a_{k}\right) \theta^{r_{1}-1}(\hat{u}) \theta^{r_{1}}\left(a_{k}^{-1}\right) \hat{w}_{1},
\end{aligned}
$$

where $t^{r_{0}} \widehat{w}_{0}=w_{0}, t^{r} \hat{u}=u$ and $t^{r_{1}} \widehat{w}_{1}=w_{1}$ in $\Psi_{k}$, as before. The first and last letters of $\theta^{r+r_{1}}\left(a_{k}\right) \theta^{r_{1}-1}(\hat{u}) \theta^{r_{1}}\left(a_{k}^{-1}\right)$ are $a_{k}$ and $a_{k}^{-1}$ which cancel, so this subword must freely reduce to the empty word. So $\theta^{r}\left(a_{k}\right) \theta^{-1}(\hat{u}) a_{k}^{-1}$ also freely reduces to the empty word, that is, $\theta^{r+1}\left(a_{k}\right) \hat{u}$ freely equals $a_{k} a_{k-1}$.

If $r=0$ then this says that $\hat{u}$ freely equals the empty word and, as before, we have a contradiction. Suppose that $r>0$. Then $\hat{u}^{-1}=\left(a_{k} a_{k-1}\right)^{-1} \theta^{r+1}\left(a_{k}\right)$ would be a positive word on $a_{0}, \ldots, a_{k-1}$ were we to remove all the $b_{1}^{ \pm 1}, \ldots, b_{l}^{ \pm 1}$ it contains. So, as $\hat{u}^{-1} t^{-r}=u^{-1}$, Corollary 4.10 applies and tells us that $u^{-1}$ would be a positive word were we to remove all the $b_{1}^{ \pm 1}, \ldots, b_{l}^{ \pm 1}$ it contains. But $r$ is the exponent sum of the $\left(a_{0} t\right)^{ \pm 1}, \ldots,\left(a_{k-1} t\right)^{ \pm 1}$ in $u$, and so we deduce the contradiction $r \leq 0$. Finally we note that the case $r<0$ also leads to a contradiction because if we replace $w$ by $w^{-1}$ it becomes the case $r>0$. 
4.4. Conclusion. We deduce from Proposition 4.8 that the word posited in Proposition 4.6 is the unique reduced word on $a_{0} t, \ldots, a_{k} t, b_{1}, \ldots, b_{l}$ that equals $a_{k}^{n} a_{2} t a_{1} a_{2}^{-1} a_{k}^{-n}$ in $\Psi_{k}$. This establishes that $\operatorname{Dist}_{\Lambda_{k}}^{\Psi_{k}} \succeq \mathscr{H}_{k}$ for all $k \geq 2$. So, by Proposition 1.2 in [6], which says that $\mathscr{H}_{k} \simeq A_{k}$ for all $k \geq 1$, we have Dist ${ }_{\Lambda_{k}} \succeq A_{k}$ for all $k \geq 2$. Added to Proposition 4.7, this completes the proof of Proposition 4.1.

Proposition 4.1 applies to the subgroup

$$
\left\langle a_{0} t, \ldots, a_{k} t, b_{1}, \ldots, b_{8}, c_{1}, \ldots, c_{8}, d\right\rangle
$$

of $\Gamma_{k}$ (presented as $Q_{k}$ of Section 2) and so, as we established $\Gamma_{k}$ to be hyperbolic in Section 3, Theorem 1.1 is proved.

\section{References}

[1] G. N. Arzhantseva and D. V. Osin, Solvable groups with polynomial Dehn functions. Trans. Amer. Math. Soc. 354 (2002), 3329-3348. Zbl 0998.20040 MR 1897402

[2] J. Barnard and N. Brady, Distortion of surface groups in CAT(0) free-by-cyclic groups. Geom. Dedicata 120 (2006), 119-139. Zbl 1167.20024 MR 2252898

[3] J. Barnard, N. Brady, and P. Dani, Super-exponential distortion of subgroups of CAT(-1) groups. Algebr. Geom. Topol. 7 (2007), 301-308. Zbl 1187.20055 MR 2308946

[4] M. R. Bridson, On the existence of flat planes in spaces of non-positive curvature. Proc. Amer. Math. Soc. 123 (1995), 223-235. Zbl 0840.53033 MR 1273477

[5] M. R. Bridson and A. Haefliger, Metric spaces of non-positive curvature. Grundlehren Math. Wiss. 319, Springer-Verlag, Berlin 1999. Zbl 0988.53001 MR 1744486

[6] W. Dison and T. Riley, Hydra groups. Comment. Math. Helv. 88 (2013), 507-540. Zbl 06216008 MR 3093501

[7] B. Farb, The extrinsic geometry of subgroups and the generalized word problem. Proc. London Math. Soc. (3) 68 (1994), 577-593. Zbl 0816.20032 MR 1262309

[8] M. Gromov, Hyperbolic groups. In Essays in group theory, Math. Sci. Res. Inst. Publ. 8, Springer-Verlag, New York 1987, 75-263. Zbl 0634.20015 MR 0919829

[9] M. Gromov, Geometric group theory (Sussex, 1991), vol. 2: Asymptotic invariants of infinite groups. London Math. Soc. Lecture Note Ser. 182, Cambridge University Press, Cambridge 1993. Zbl 0841.20039 MR 1253544

[10] J. Howie, On the asphericity of ribbon disc complements. Trans. Amer. Math. Soc. 289 (1985), 281-302. Zbl 0572.57001 MR 779064

[11] T. Mecham and A. Mukherjee, Hyperbolic groups which fiber in infinitely many ways. Algebr. Geom. Topol. 9 (2009), 2101-2120. Zbl 1223.20036 MR 2551664

[12] M. Mitra, Cannon-Thurston maps for trees of hyperbolic metric spaces. J. Differential Geom. 48 (1998), 135-164. Zbl 0906.20023 MR 1622603

[13] M. Mitra, Coarse extrinsic geometry: a survey. In The Epstein birthday schrift, Geom. Topol. Monogr. 1, Geom. Topol. Publ., Coventry 1998, 341-364. Zbl 0914.20034 MR 1668308 
[14] Ch. Pittet, Géométrie des groupes, inégalités isopérimétriques de dimension 2 et distorsions. PhD thesis, Université de Genève, Genève 1992.

[15] H. E. Rose, Subrecursion: functions and hierarchies. Oxford Logic Guides 9, Oxford University Press, Oxford 1984. Zbl 0539.03018 MR 752696

Received September 22, 2011; revised June 5, 2012

N. Brady, Department of Mathematics, Physical Sciences Center, 601 Elm Ave, University of Oklahoma, Norman, OK 73019, U.S.A.

E-mail: nbrady@math.ou.edu

W. Dison, Bank of England, Threadneedle Street, London, EC2R 8AH, UK

E-mail: william.dison@gmail.com

T. R. Riley, Department of Mathematics, 310 Malott Hall, Cornell University, Ithaca, NY 14853, U.S.A.

E-mail: tim.riley@math.cornell.edu 